\title{
A Biophysical Model of Decision Making in an Antisaccade Task Through Variable Climbing Activity
}

\author{
Vassilis Cutsuridis ${ }^{1, *}$, Ioannis Kahramanoglou ${ }^{1}$, Stavros Perantonis ${ }^{1}$, \\ Ioannis Evdokimidis ${ }^{2}$, and Nikolaos Smyrnis ${ }^{2,3}$ \\ ${ }^{1}$ Computational Intelligence Laboratory, Institute of Informatics and Telecommunications, \\ National Center for Scientific Research "Demokritos", Agia Paraskevi, Athens GR-15310 \\ \{vcut, ikahra, sper\}@iit.demokritos.gr \\ ${ }^{2}$ Cognition and Action Group, Neurology Department, National University of Athens, \\ Aeginition Hospital, 72 Vas Sofias Ave, Athens GR-11528 \\ ${ }^{3}$ Psychiatry Department, National University of Athens, Aeginition Hospital, \\ 72 Vas Sofias Ave, Athens GR-11528 \\ smyrnis@med.uoa.gr
}

\begin{abstract}
We present a biophysical model of saccade initiation based on competitive integration of planned and reactive cortical saccade decision signals in the intermediate layer of the superior colliculus. In the model, the variable slopes of the climbing activities of the input cortical decision signals are produced from variability in the conductances of $\mathrm{Na}^{+}, \mathrm{K}^{+}, \mathrm{Ca}^{2+}$ activated $\mathrm{K}^{+}$, NMDA and GABA currents. These cortical decision signals are integrated in the activities of buildup neurons in the intermediate layer of the superior colliculus, whose activities grow nonlinearly towards a preset criterion level. When the level is crossed, a movement is initiated. The resultant model reproduces the unimodal distributions of saccade reaction times (SRTs) for correct antisaccades and erroneous prosaccades as well as the variability of SRTs (ranging from $80 \mathrm{~ms}$ to $600 \mathrm{~ms}$ ) and the overall $25 \%$ of erroneous prosaccade responses in a large sample of 2006 young men performing an antisaccade task.
\end{abstract}

\section{Introduction}

In the brain, climbing activity is a prominent profile of neuronal activity observed in the thalamus, superior colliculus, primary motor cortex, prefrontal cortex and other brain areas and it is found to be related to the anticipation of forthcoming events and to the generation of movements. Climbing activity spans from hundreds of milliseconds up to tens of seconds [1]. In the frontal eye fields of monkeys there are populations of visuomotor neurons that begin to fire in advance of saccades, with their activity rising linearly upon presentation of a suitable target stimulus [5]. Buildup cells in the monkey's superior colliculus (SC) begin to linearly build up their activity after the signal to make a saccade is presented [8]. The rate of rise varies randomly from trial to trial and the saccade is initiated when this activity reaches a fixed threshold [5], [6].

\footnotetext{
* Corresponding author.
} 
The model presented in this paper is an attempt to model the biophysical mechanisms underlying the generation of slowly varying climbing, temporal integrator-like activity of the reactive and planned input decision signals of a SC model in an antisaccade task [9]. This work combines and extends previous biophysical models [1], [9], [10].

\section{Materials and Methods}

\subsection{Basis of the Model}

In a modeling attempt of the antisaccade task [4], Cutsuridis and colleagues [9] hypothesized that the preparation of an antisaccadic eye movement consisted of two cortically independent and spatially separated decision signals representing the reactive and planned saccade signals, whose linearly rising phases are derived from two normal distributions with different means and standard deviations. These two cortical decision signals were then integrated at opposite colliculi locations, where they competed against each other via lateral excitation and remote inhibition. A saccade was initiated when these decision processes, represented by the neuronal activity of SC buildup neurons with nonlinear growth rates varying randomly from a normal distribution, gradually build up their activity until reaching a preset criterion level. The crossing of the preset criterion level in turn released the "brake" from the SC burst neurons and allowed them to discharge resulting in the initiation of an eye movement. The model's main prediction was that there is no need of a top-down inhibitory signal that prevents the error prosaccade from being expressed, thus allowing the correct antisaccade to be released. Moreover, the model offered a functional rationale at the SC neuronal population level of why the antisaccadic reaction times are so long and variable and simulated accurately the correct and error antisaccade latencies, the shape distributions and the error probabilities.

Our intention in this study is to model the biophysically plausible mechanisms that can produce climbing activity with adjustable slope. We extend the SC model by adding two cortical modules that will generate the planned and reactive decision signals. The decision signals will be derived from the population activities of networks of pyramidal neurons and inhibitory interneurons. We will use HodgkinHuxley mathematical formulations to explore the biophysical mechanisms that give rise to the randomly varying climbing activities of the cortical decision signals. These decision signals will then drive the SC model and generate correct antisaccade and error prosaccade reaction time (RT) distributions as well as response probabilities. These simulated RT distributions and error probabilities will be compared to psychophysically derived latency distributions and error probabilities [3], [7].

\subsection{Architecture}

Standard Hodgkin-Huxley modeling techniques were used to simulate networks of single compartmental models of cortical pyramidal neurons and cortical inhibitory interneurons (IN). Pyramidal neuron membrane potential obeyed 
$C d V / d t=-\left(I_{\text {leak }}+I_{N a}+I_{N a P}+I_{K S}+I_{H V A}+I_{C}+I_{D R}+I_{A H P}+I_{A M P A}+I_{N M D A}+I_{G A B A}\right)+I_{i n j}$

with $C_{m}=1 \mu \mathrm{F} \mathrm{cm}^{-2}$. GABAergic inhibitory interneuron membrane potential obeyed

$$
C d V / d t=-\left(I_{\text {leak }}+I_{N a}+I_{D R}+I_{A M P A}+I_{N M D A}+I_{G A B A}\right)+I_{i n j}
$$

with $C_{m}=1 \mu \mathrm{F} \mathrm{cm}^{-2}$. Ionic currents $\mathrm{I}_{\mathrm{Na}}, \mathrm{I}_{\mathrm{NaP}}, \mathrm{I}_{\mathrm{Ks}}, \mathrm{I}_{\mathrm{C}}, \mathrm{I}_{\mathrm{DR}}$, and $\mathrm{I}_{\mathrm{HVA}}$ were modeled as in [10], whereas $I_{\text {AHP }}$ was modeled as in [1]. Table 2 of [10] provided a summary of the gating variables and their respective powers for all ionic conductances used in this study. The synaptic currents ( $\mathrm{I}_{\mathrm{AMPA}}, \mathrm{I}_{\mathrm{NMDA}}$, and $\mathrm{I}_{\mathrm{GABA}}$ ) were given by double exponential functions exactly as in [1]. Synaptic short term dynamics were determined by the available synaptic efficacy $(\mathrm{R})$ and a utilization variable $(\mathrm{u})$ exactly as in [1]. We simulated low spontaneous background activity in the network, by delivering random noise to all pyramidal and GABAergic cells, generated from Poisson processes convolved with the AMPA, NMDA and GABA synaptic conductances. Because very little is known about the detailed connectivity of neurons and the associated synaptic strengths in the frontal cortices, we intentionally kept the network model as general as possible. Two networks of 10 pyramidal cells and 5 GABAergic interneurons each were simulated. In each network, we assumed that all pyramidal cells and GABAergic interneurons were fully connected [10]. The output of each network was the average population activity of a homogenous population of neurons with identical connections. These outputs were then used as the input drives of the superior colliculus (SC) model [9].

\subsection{Implementation}

The simulations were performed on a Pentium IV $3.2 \mathrm{GHz}$ PC with MATLAB's version R13 installed. The whole system of differential and algebraic equations was implemented in MATLAB (The MathWorks, Inc, Natick, MA). The differential equations of the cortical neural integrator model were integrated numerically using one of the MATLAB ordinary differential equation solvers (mainly ode23s, a one step solver based on modified Rosenbrock formula of order 2 [2]) with time step $\Delta t=$ $0.001 \mathrm{~ms}$. The differential equations of the SC model were integrated numerically using one of the MATLAB ordinary differential equations solvers (ode45, an implicit solver based on the Dormand-Prince pair method [2]) with time step $\Delta t=0.001 \mathrm{~ms}$ ). Relative (error) tolerance was set to $10^{-6}$.

\section{Experiments and Results}

\subsection{Experimental Setup}

The data used in this study were collected in an antisaccade task [3], [7]. Details of the experimental procedure used for the collection of these data are described therein [3], [7]. Briefly, 2006 conscripts of the Greek Air Force were instructed to perform eye movements in the opposite direction from the location of a stimulus that appears in their right or left peripheral visual field while they are fixating on a central 
stimulus. The correct or error saccade reaction time (SRT) was measured in each trial for every subject. Trials with reaction times $<80 \mathrm{~ms}$ were excluded as anticipations and trials with reaction times $>600 \mathrm{~ms}$ were excluded as no response trials. The median RT and the inter-quartile range for antisaccades and error prosaccades of all 2006 conscripts were grouped into ten virtual groups after performing clustering analysis using the STATISTICA software version 5.5 (StatSoft, Inc, Tulsa, OK). The purpose of the cluster analysis was to partition the observations into groups ("clusters") so that the pairwise dissimilarities between those assigned to the same cluster tend to be smaller than those in a different cluster. We demonstrate below the results from all ten clusters.

\subsection{Results}

The observed variability in the rising phase (slope) of the average firing rates of the pyramidal neurons was found to be due to noise in the conductances of the $\mathrm{I}_{\mathrm{NaP}}$ and $\mathrm{I}_{\mathrm{NMDA}}$ currents. Noise in the conductances of $\mathrm{I}_{\mathrm{Ks}}, \mathrm{I}_{\mathrm{DR}}, \mathrm{I}_{\mathrm{C}}$ and $\mathrm{I}_{\mathrm{HVA}}$ currents didn't produce any variability in the rising phase of the average firing rate. The slope of the climbing activity was carefully adjusted so that the simulated correct and error RT distributions and the error probabilities to approximate the experimental ones in an antisaccade task (see Table 1). We estimated the slopes of the rising phases of the average firing rates of two cortical networks of neurons in each trial by fitting to them a straight line. We used these slope values as values of the slopes of the rising phases of the planned and reactive inputs of [9]. The slope values of the reactive and planned inputs were sorted in ascending order, so that the slope of the reactive input was always greater than the slope of the planned input. The threshold was adjusted, so that the simulated error rate closely matched the observed. Its value was set to a different value for each group, but it was kept fixed across trials for each group [9].

Table 1. (Columns 2-4) Simulated correct median, error median, and error rate for average and all ten groups. Values in parentheses stand for experimental values. Units: correct SRT (ms), error SRT (ms). (Columns 5-6) Values of $\chi^{2}$ test of homogeneity between correct and error experimental and simulated percent density distributions for antisaccades and error prosaccades. $\chi^{2}$ values marked with an asterisk indicate a significant difference between the simulated and the observed RT distributions. Rejection region: $\chi^{2} \geq \chi_{0.05}^{2}$ (37.65). The degrees of freedom were 25 .

\begin{tabular}{lccccc}
\hline & $\begin{array}{c}\text { Median } \\
\text { RT } \\
\text { of antisaccades }\end{array}$ & $\begin{array}{c}\text { Median } \\
\text { RT of error } \\
\text { prosaccades }\end{array}$ & $\begin{array}{c}\text { \% antisaccade } \\
\text { error rate }\end{array}$ & $\begin{array}{c}\text { antisaccade } \boldsymbol{\chi}^{\mathbf{2}} \\
\text { value }\end{array}$ & $\begin{array}{c}\text { prosaccade } \\
\boldsymbol{\chi}^{\mathbf{2}} \text { value }\end{array}$ \\
\hline G 1 & $254.80(242.40)$ & $212.99(216.66)$ & $24.27(17.02)$ & 35.21 & 24.18 \\
G 2 & $282.38(288.44)$ & $188.10(193.66)$ & $23.93(28.86)$ & 31.82 & 27.97 \\
G 3 & $263.10(251.79)$ & $180.63(175.53)$ & $20.87(24.79)$ & 30.34 & 21.82 \\
G 4 & $365.69(349.42)$ & $218.99(221.36)$ & $37.00(34.58)$ & 36.46 & 35.67 \\
G 5 & $218.20(213.58)$ & $177.85(172.77)$ & $27.36(24.92)$ & 35.21 & 24.18 \\
G 6 & $294.174(288.16)$ & $279.541(265.20)$ & $13.04(16.15)$ & 36.15 & 34.92 \\
G 7 & $276.50(279.21)$ & $202.97(201.96)$ & $38.62(39.07)$ & $90.5^{*}$ & 32.56 \\
G 8 & $281.89(280.91)$ & $212.54(201.92)$ & $20.15(23.73)$ & 32.16 & $96.24^{*}$ \\
G 9 & $251.30(249.27)$ & $209.90(211.65)$ & $12.41(12.02)$ & $56.06^{*}$ & $83.57^{*}$ \\
G 10 & $327.56(307.5)$ & $331.07(326.99)$ & $20.05(21.81)$ & 33.88 &
\end{tabular}


The SC model was allowed to run for 1000 trials in each group. We recorded the simulated median antisaccade and error prosaccade RT values and the error rates for each group (see Table 1). In order for each group to compare the SRT distributions of the real experimental data with the simulated SRT distributions, we normalized the SRT distribution of each subject data and then added the normalized distributions for all subjects belonging to the same group. More specifically, the time interval between the $80 \mathrm{~ms}$ and $600 \mathrm{~ms}$ was divided into twenty-six categories, each lasting $20 \mathrm{~ms}$ (e.g. category 1 was between $80 \mathrm{~ms}$ and $100 \mathrm{~ms}$, category 2 between $100 \mathrm{~ms}$ and $120 \mathrm{~ms}$, and so forth). For each category we calculated its percent relative frequency of response times. The mean frequency for all subjects in a group was then calculated. The discrepancy in each category between the simulated and experimental correct and error distributions was measured by the squared difference between the observed (simulated) and the expected (experimental) frequencies divided by the expected frequency ((Observed - Expected $)^{2} /$ Expected). The $\chi^{2}$ value was the sum of these quantities for all categories. The rejection region was set at $\chi^{2} \geq \chi_{0.05}^{2}$. The $\chi^{2}$ test of homogeneity tested the null hypothesis of whether the simulated and experimental normalized distributions of SRTs for antisaccades and error prosaccades differ between them and showed a significant difference in 2 of the 10 comparisons for antisaccade RT distributions and 2 of the 10 comparisons for the error prosaccade RT distributions (see Table 1).

\section{Conclusion}

The simulations of the model presented here show that the randomly varying climbing activities of the input decision signals of a SC model in an antisaccade task are due to the interplay of $\mathrm{K}^{+}, \mathrm{Na}^{+}$, and $\mathrm{Ca}^{2+}$ activated $\mathrm{K}^{+}$currents as well as due to variability of NMDA synaptic currents. The model is successful at predicting the correct antisaccade and error prosaccade RT distributions as well as the response probabilities from a population of 2006 subjects. There are further paradigms and architectures to which this model can be extended to. For instance, in this study we assumed that the internal properties of all pyramidal neurons in the network were the same (homogenous) and that the connectivity was symmetric. However, real populations of neurons will always have a certain degree of heterogeneity in their internal parameters and in their connectivity patterns. For this reason, we are in the process of examining other more realistic cases of neuronal connectivity in our network. Finally, we are investigating in a more systematic way which ionic conductances have the strongest effects on the rising phase of the average firing rate of the pyramidal neurons and what are the mechanisms that cause the variability in the climbing activities.

\section{References}

1. Durstewitz, D.: Self Organizing Neural Integrator Predicts Interval Times Through Climbing Activity. J. Neurosci. 23(12) (2003) 5342-5353

2. Shampine, L.F., Reichelt, M.W.: The MATLAB ODE Suite. SIAM Journal on Scientific Computing. 18 (1997) 1-22 
3. Evdokimidis, I., Smyrnis, N., Constantinidis, T.S., Stefanis, N.C., Avramopoulos, D., Paximadis, C., Theleritis, C., Efstratiadis, C., Kastrinakis, G., Stefanis, C.N.: The Antisaccade Task in a Sample of 2006 Young Men I. Normal Population Characteristics. Exp Brain Res. 147 (2002) 45-52

4. Hallett, P.R.: Primary and Secondary Saccades to Goals Defined by Instructions. Vis. Res. 18 (1978) 1279-1296

5. Hanes, D.P., Schall, J.D.: Neural Control of Voluntary Movement Initiation. Science. 274 (1996) 427-430

6. Reddi, B.A.J., Carpenter, R.H.S.: The Influence of Urgency on Decision Time. Nat. Neurosci. 3 (2000) 827-831

7. Smyrnis, N., Evdokimidis, I., Stefanis, N.C., Constantinidis, T.S., Avramopoulos, D., Theleritis, C., Paximadis, C., Efstratiadis, C., Kastrinakis, G., Stefanis, C.N.: The Antisaccade Task in a Sample of 2006 Young Males II. Effects of Task Parameters. Exp. Brain Res. 147 (2002) 53-63

8. Everling, S., Dorris, M.C., Klein, R.M., Munoz, D.P.: Role of Primate Superior Colliculus in Preparation and Execution of Anti-saccades and Prosaccades. J. Neurosci. 19(7) (1998) 2740-2754

9. Cutsuridis, V., Smyrnis, N., Evdokimidis, I., Perantonis, S.: A Neural Model of Decision Making by the Superior Colliculus in an Antisaccade Task. submitted to Biol. Cybernetics

10. Durstewitz, D., Seamans, J.K., Sejnowski, T.J.: Dopamine-Mediated Stabilization of Delay-Period Activity in a Network Model of Prefrontal Cortex. J. Neurophys. 83 (2000) 1733-1750 